Editorial

\title{
A simple method for isolation of rest of trypsinized stem cells with magnetic beads
}

\section{Sudhir Bhatia}

GENEKAM Biotechnology AG, Germany

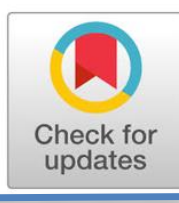

It is a a common practice that human stem cells like mesenchymal stromal stem cells (MSC) can be isolated from different sources like umbilical cord tissue, placenta, adipose tissue and bone marrow as these are multipotent cells, which can differentiate into different kinds of cells like adipocytes, osteocytes, chondrocytes and so on. ${ }^{1}$ During the isolation, the passaging of the cells is needed to get the required minimum number of stem cells for experimental and therapeutic applications. As the stem cells grow very slowly, therefore their needed number may be many times difficult to obtain. The stromal stem cells are adherent to the plastic surface of cell culture flasks during their selection and growth, hence these cells are obtained through the trypsin treatment because such treatment will detach them from the surface. Trypsin treatment is one of the common solutions used in laboratories around the world. After the trypsin treatment, the user collects the solution containing detached cells in a tube and they are centrifuged to get a pellet for further applications. MSC has different markers e.g. CD90, CD105, CD73 and they are negative for CD34, HLA DR, CD45 depending upon the source of isolation. Usually, the user disposes of the cell culture flasks as waste after trypsin treatment. Till now, there is no laboratory in the world, which makes thoughts that there may be still stem cells remaining in the flasks after the trypsin treatment. The user checks the presence of stem cells during trypsinization with the microscope whether the whole population of stem cells has been collected. Magnetic beads are being used around the world to isolate different kinds of cells like CD4, CD8, CD34, and other cells. One of the advantages of magnetic isolation is that they can be used to isolate cells from solutions containing even a low number of targeted cells. We conducted the experiments to see whether all remaining MSC can be obtained from trypsin treated flask with magnetic beads isolation.

Human Umbilical cord tissue was cut into $1 \mathrm{~mm}$ sized small pieces and placed in flasks to get MSC in DMEM (Lonza) containing 1\% Streptopenicillin antibiotics (Biochrome) and $10 \%$ fetal calf serum (Biochrome). On the $7^{\text {th }}$ day, the growth of the stem cells was observed under a microscope. The MSC after reaching $80 \%$ confluency were trypsinized with trypsin solution (Biochrome) and they were processed to get passage 1 (P1) in a new flask in PBS (Biochrome) after obtaining the pellet through centrifugation. The P1 stem cells were grown for the next 8 days to harvest with trypsin solution to get passage P2. The $15 \mathrm{ml}$ PBS was added to the trypsinized flask (which should be without any cells) and observed under a microscope to see whether they are still the rest of the cells.

Corresponding author

E-mail address:anfrage@genekam.de (Sudhir Bathia) 
It was found that there are no cells in the flask under the microscope. This $15 \mathrm{ml}$ PBS was collected in a $15 \mathrm{ml}$ tube. To it, $50 \mathrm{ul}$ of magnetic beads specific for CD90 (Genekam) were added. This was kept at room temperature for 30 minutes. After that, the beads were removed in a magnetic rack (Genekam). The removed magnetic beads were suspended in $5 \mathrm{ml}$ of DMEM solution and they were observed under a microscope. After that, the cells were cultured and observed under a microscope for further growth for 7-10 days.

Stem cells grew out of small pieces of umbilical tissue in flasks around 6$9^{\text {th }}$ day as adherent cells on the plastic bottom of flasks. They were trypsinized to obtain successfully a pellet for passage 1. First passage P1 was done successfully. The stem cells show their typical structure under the microscope. Under the microscope after trypsin treatment to get second passage P2, we were unable to see any cell in $15 \mathrm{ml}$ PBS from trypsinized flasks. During the magnetic isolation with CD90 specific Magnetic beads, it was found under the microscope that there are huge numbers of cells being attached to beads. These cells are subcultured and they were grown in MSC cells (Picture 1), where the magnetic beads are attaching to parts of cells. These cells were used for further passage and gave new stem cells, which were adherent to plastic. The results indicate that there are still a huge number of rest cells in the trypsinized flask but most of the users think that they have isolated all cells, which is not true and they are throwing away valuable stem cells. The magnetic beads can be used to isolate the rest of MSC because for many applications, there is a need of the largest number of stem cells for conducting studies like flow cytometry, molecular testing along with pre-clinical and clinical studies. The magnetic beads method is simple and can be performed within a short period. It does not need any extra expensive instruments except a magnetic rack (Picture 2). Such a magnetic rack can be used for a very long time and for different cells to be isolated. There are a large number of remaining stem cells after the trypsin treatment in the flasks and they can be successfully removed with CD90 magnetic breads for further culturing.

\section{DISCLOSURE STATEMENT}

The author declare that they have no conflict of interest.

\section{FUNDING INFORMATION}

The author declared that this case has received no financial support.

\section{REFERENCES}

1. Atala A, Lanza R, Thomson JA, Nerem R. Principles of Regenerative Medicine. Elsevier; 2011. doi:10.1016/C2009-0-61040-0.

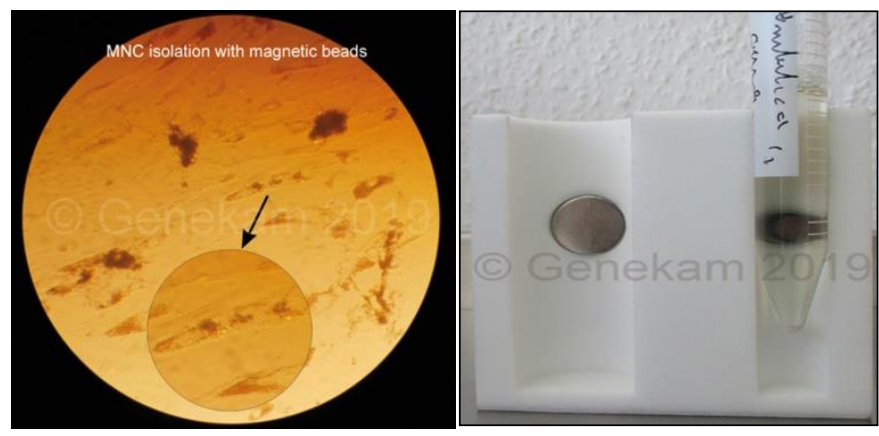

Picture 1

Picture 2 